\title{
Optical-conductivity sum rule in cuprates and unconventional charge density waves: a short review
}

\author{
L. Benfatto ${ }^{1}$ and S.G. Sharapov ${ }^{2}$ \\ ${ }^{1} S M C-I N F M-C N R$ and Department of Physics, \\ University of Rome "La Sapienza", \\ Piazzale Aldo Moro 5, 00185, Rome, Italy \\ ${ }^{2}$ Department of Physics and Astronomy, \\ McMaster University, Hamilton, \\ Ontario, Canada, L8S $4 M 1$
}

(Dated: October 30, 2018)

\begin{abstract}
We begin with an overview of the experimental results for the temperature and doping dependences of the optical-conductivity spectral weight in cuprate superconductors across the whole phase diagram. Then we discuss recent attempts to explain the observed behavior of the spectral weight using reduced and full models with unconventional $d_{x^{2}-y^{2}}$ charge-density waves.

PACS numbers: 71.10.-w, 74.25.Gz, 74.72.-h
\end{abstract}

\section{PRELIMINARIES}

The optical-conductivity sum rule plays an important role in the understanding of the physics of cuprate superconductors. The behavior of the spectral weight in the pseudogap phase and when the system undergoes a superconducting transition has been the object of the experimental investigation in the last few years [1-8]. Considering that the analysis of the experimental data and the extraction of the spectral weight involves a lot of theoretical assumptions, so that even opposite conclusions can be drawn based on the same data $[5,9,10]$, we decided to describe the current experimental situation in the introductory Sec. II using the theoretical language from the very beginning, to avoid any possible confusion. We find that a clear perception of these assumptions is crucial for the correct interpretation of the experiments.

In Sec. III we briefly summarize the recent history of unconventional density waves, and introduce the reduced $d$-density wave (DDW) model, which can be regarded as the charge density wave equivalent of the reduced BCS model. Then based on Refs. [11, 12] we discuss the optical conductivity and the sum rule for the reduced model, as an attempt to interpret some of the recent data on the sum rule of cuprates. In Sec. IV we discuss the limitations of the reduced model, as already pointed out clearly in Ref. [12], and consider the recent results obtained for a more generic microscopic Hamiltonian by Aristov and Zeyher [13]. Some of the remaining questions and the relationship to other systems are discussed in Sec. V.

\section{GENERAL NOTATIONS AND OVERVIEW OF EXPERIMENTS}

\section{A. Optical conductivity and sum rule for it}

The optical-conductivity sum rule is intimately related to the general properties of the current-current response function $[14,15]$. Since we are interested in describing lattice systems, let us consider a general case of the electronic system described by the Hamiltonian:

$$
H=H_{0}+H_{i n t}, \quad H_{0}=-\sum_{i j} t_{i j} c_{i \sigma}^{\dagger} c_{j \sigma}-\mu \sum_{i} c_{i \sigma}^{\dagger} c_{i \sigma},
$$

where the field operator $c_{i \sigma}^{\dagger}$ creates an electron of spin $\sigma$ at the site $\mathbf{r}_{i}, t_{i j}$ is the hopping parameter, $\mu$ is the chemical potential and $H_{i n t}$ is the interaction term. For the kinetic part $H_{0}$ a proper description of the cuprates is obtained by restricting the sum to next-neighboring sites on a squared lattice that results in a band dispersion $\varepsilon(\mathbf{k})=-2 t\left(\cos k_{x} a+\cos k_{y} a\right)+4 t^{\prime} \cos k_{x} a \cos k_{y} a$, where $a$ is the lattice constant. However, to avoid unnecessary complications we will also refer below to the simplest case $t^{\prime}=0$. Throughout the paper units $\hbar=k_{B}=c=1$ are chosen.

Given the model (2.1), the definitions of the particle current $\mathbf{j}\left(\mathbf{r}_{i}\right)$ and the so-called diamagnetic tensor $\tau_{i i}\left(\mathbf{r}_{i}\right)$ follow from the first- and second-order derivatives of the Hamiltonian $H(\mathbf{A})$ with respect to the external vector potential $\mathbf{A}$ 
$[16]:$

$$
H\left(A_{i}\right) \approx H(0)-\sum_{j}\left[e A_{i}\left(\mathbf{r}_{\mathbf{j}}\right) j_{i}\left(\mathbf{r}_{\mathbf{j}}\right)-\frac{e^{2}}{2} A_{i}^{2}\left(\mathbf{r}_{\mathbf{j}}\right) \tau_{i i}\left(\mathbf{r}_{\mathbf{j}}\right)\right],
$$

so that the total electric current density is expressed as

$$
J_{i}(\mathbf{r})=-\delta H / \delta A_{i}(\mathbf{r})=e j_{i}(\mathbf{r})-e^{2} \tau_{i i}(\mathbf{r}) A_{i}(\mathbf{r}),
$$

By using the generalized notation $j_{\mu}=\left(j_{i}, j_{0}\right)$ where the index $\mu=(i, 0)$ with $i=1,2$ indicates spatial and time components respectively, and $j_{0}$ is the particle density, one can evaluate $\left\langle J_{\mu}(q)\right\rangle$ within linear response theory $[15-17]$ and obtain $J_{\mu}(q)=e^{2} K_{\mu \nu}(q) A_{\nu}(q)$, where the electromagnetic kernel $K_{\mu \nu}$ is defined as:

$$
K_{\mu \nu}\left(\mathbf{q}, i \Omega_{m}\right)=-\tau_{\mu \mu} \delta_{\mu \nu}\left(1-\delta_{\nu 0}\right)+\Pi_{\mu \nu}\left(\mathbf{q}, i \Omega_{m}\right) .
$$

Here $\Pi_{\mu \nu}\left(\mathbf{q}, i \Omega_{m}\right)$ is the current-current correlation function

$$
\Pi_{\mu \nu}\left(\mathbf{q}, i \Omega_{m}\right)=\frac{1}{N} \int_{0}^{\beta} d \tau e^{i \Omega_{m} \tau}\left\langle T_{\tau} j_{\mu}(\mathbf{q}, \tau) j_{\nu}(-\mathbf{q}, 0)\right\rangle,
$$

$\tau$ is the imaginary time, $\beta=1 / T, \Omega_{m}=2 \pi m T$ is the bosonic Matsubara frequency and $N$ is the number of unit cells. Finally, using that $\mathbf{A}(\omega)=\mathbf{E}(\omega) / i(\omega+i 0)$, where $\mathbf{E}$ is the electric field, one arrives at the famous Kubo formula

$$
\sigma(\omega)=-i e^{2} \frac{K_{i i}(\mathbf{q}=0, \omega)}{V(\omega+i 0)}=i e^{2} \frac{<\tau_{i i}>-\Pi_{i i}(\mathbf{q}=0, \omega)}{V(\omega+i 0)},
$$

where $V$ is the unit-cell volume and the standard analytic continuation $i \Omega_{m} \rightarrow \omega+i 0$ was made. To avoid confusion, we will indicate the imaginary bosonic frequencies with $i \Omega_{m}$, the imaginary fermionic frequencies with $i \omega_{n}=i \pi(2 n+1) T$ and the real frequencies with $\omega$. Since an isotropic system is considered we can omit the index $i$ as done in the LHS of Eq. (2.6) and in what follows.

To extract the real and imaginary parts of the optical conductivity defined in Eq. (2.6) we should consider the effect of disorder. Indeed, for the (ideal) case of a system without disorder by taking the real part of (2.6) one obtains $\operatorname{Re} \sigma(\omega)=\left(\pi e^{2} / V\right) \delta(\omega)[<\tau>-\operatorname{Re} \Pi(\mathbf{0}, \omega)]+\left(e^{2} / V\right) \operatorname{Im} \Pi(\mathbf{0}, \omega) / \omega$. The presence of a delta function, which means an ideal conductivity of the system, is an artifact of the assumption that no impurities are present. As soon as disorder is present one expects that in the normal state the following identity holds:

$$
\operatorname{Re} \Pi(\mathbf{q}=0, \omega \rightarrow 0)=<\tau>,
$$

so that only the regular part of $\sigma(\omega)$ survives, defined as usual as:

$$
\operatorname{Re} \sigma(\omega)=\frac{e^{2}}{V} \frac{\operatorname{Im} \Pi(\mathbf{q}=\mathbf{0}, \omega)}{\omega} .
$$

Thus, using the Kramers-Kronig $(\mathrm{KK})$ relations for $\Pi(\mathbf{q}=0, \omega)$ one can derive the well-know sum rule:

$$
W(T)=\int_{-\infty}^{\infty} \operatorname{Re} \sigma(\omega) d \omega=\frac{e^{2}}{V} \int_{-\infty}^{\infty} \frac{\operatorname{Im} \Pi(\mathbf{q}=\mathbf{0}, \omega)}{\omega} d \omega=\frac{\pi e^{2}}{V} \operatorname{Re} \Pi(\mathbf{q}=\mathbf{0}, \omega=0)=\frac{\pi e^{2}}{V}<\tau>.
$$

Observe that Eq. (2.7) and (2.9) require that for the system in the presence of disorder, the dynamic $(\mathbf{q}=0, \omega \rightarrow 0)$ and static $(\omega=0, \mathbf{q} \rightarrow 0)$ limits of $\operatorname{Re} \Pi(\mathbf{q}, \omega)$ commute. This is indeed the case for a disordered system, but not for a clean one, where usually $\operatorname{Re} \Pi(\mathbf{0}, \omega)$ vanishes as $\omega \rightarrow 0[16,17]$.

As one can see from Eq. (2.9) the sum rule is directly defined by the diamagnetic tensor, which in turn depends, according to Eq. (2.2), on the way how the vector potential $\mathbf{A}$ enters the Hamiltonian of the system. When a continuum model is considered instead of Eq. (2.1), the kinetic term is expressed as $\int(-\nabla)^{2} / 2 m$ and $\mathbf{A}$ is inserted using the minimal coupling prescription $-i \nabla \rightarrow-i \nabla-e \mathbf{A}$. For lattice systems the equivalent of the minimal coupling prescription is the so-called Peierls ansatz [14, 16, 18], which corresponds to inserting the gauge field $\mathbf{A}$ in Eq. (2.1) by means of the substitution $c_{i} \rightarrow c_{i} e^{-i e \int \mathbf{A} \cdot d \mathbf{r}}$. In this case, it is clear that when the interaction term of the Hamiltonian is a density-density interaction, as in Eq. (3.2) below, only the kinetic hopping term is modified, while the interaction 
term is gauge invariant (GI). As a result, the current/density operator and the diamagnetic tensor can be expressed (for small $\mathbf{q}$ ) as:

$$
\begin{aligned}
j_{\mu}(\mathbf{q}, t) & =\frac{1}{N} \sum_{\mathbf{k}, \sigma} v_{\mu}(\mathbf{k}) c_{\mathbf{k}-\mathbf{q} / 2 \sigma}^{\dagger} c_{\mathbf{k}+\mathbf{q} / 2 \sigma} \\
\left\langle\tau_{i i}\right\rangle & =\frac{1}{N} \sum_{\mathbf{k}, \sigma} \frac{\partial^{2} \varepsilon_{\mathbf{k}}}{\partial k_{i}^{2}} n_{\mathbf{k}, \sigma}
\end{aligned}
$$

where

$$
v_{\mu}(\mathbf{k})=\left(v_{\mathbf{k}}^{F}, 1\right)
$$

and $\left(v_{\mathbf{k}}^{F}\right)_{i}=\partial \varepsilon_{\mathbf{k}} / \partial k_{i}$ is the Fermi velocity. As a consequence, we obtain the restricted optical-conductivity sum rule:

$$
W\left(\omega_{M}, T\right)=\int_{-\omega_{M}}^{\omega_{M}} \operatorname{Re} \sigma_{i i}(\omega, T) d \omega \equiv W(T)=\frac{\pi e^{2}}{V}\left\langle\tau_{i i}\right\rangle=\frac{\pi e^{2}}{V N} \sum_{\mathbf{k}, \sigma} \frac{\partial^{2} \varepsilon_{\mathbf{k}}}{\partial k_{i}^{2}} n_{\mathbf{k}, \sigma}=-\frac{\pi e^{2} a^{2}}{V} \frac{\langle K\rangle}{n_{d}},
$$

where the last equality, relating the spectral weight to the average value of the kinetic energy $K$, only holds for a nearest-neighbor tight-binding model $\left(t^{\prime}=0\right)$, and $n_{d}$ is the dimensionality of the system. Observe also that here $K$ includes the sum over two spin components, so that the factor $1 / n_{d}$ comes from the fact that $\sum_{\mathbf{k}} \partial^{2} \varepsilon_{\mathbf{k}} / \partial k_{i}^{2}=$ $\sum_{\mathbf{k}} 2 t a^{2} \cos k_{i} a=-\left(a^{2} / n_{d}\right) \sum_{\mathbf{k}} \varepsilon_{\mathbf{k}}$. In writing Eq. (2.13) we introduced a cut-off $\omega_{M}$ in the frequency integration to make contact with the experiments, where only a limited range of frequency is accessible. However, the equivalence we established between Eq. (2.9) and Eq. (2.13) relies on the fact that even though in deriving Eq. (2.9) an integration up to an infinite cut-off is formally required, an intrinsic cut-off is provided by the energy $\omega_{M}$ below which the tight-binding description (2.1) is valid. As we shall see, the definition of such a cut-off in cuprates is a quite delicate issue. Indeed, it is clear from Eq. (2.13) that the spectral weight depends both on temperature and interaction trough the occupation number $n_{\mathbf{k}, \sigma}$, and as a consequence it is a powerful tool to test theoretical predictions coming from different models. This behavior should be contrasted to the case of an electronic system described by a quadratic band dispersion $\varepsilon_{\mathbf{k}}=\mathbf{k}^{2} / 2 m$. Indeed, in this case the tensor $\left\langle\tau_{i j}\right\rangle$ reduces to $n \delta_{i j} / m$, where $n$ is the total carrier density, so that Eq. (2.9) reduces to the so-called f-sum rule

$$
\int_{-\infty}^{\infty} \operatorname{Re} \sigma(\omega) d \omega=\frac{\pi n e^{2}}{m}
$$

which is temperature and interaction independent. To make contact between the restricted and full f-sum rule it can be useful to define the frequency-dependent spectral weight:

$$
W(\omega, T)=\int_{-\omega}^{\omega} \operatorname{Re} \sigma\left(\omega^{\prime}, T\right) d \omega^{\prime}
$$

In a lattice system one would expect that below $\omega=\omega_{M}$ only transitions within the conduction band are included, while at higher energies interband processes play also a role,

$$
\sigma(\omega)=\sigma_{\text {intra }}(\omega)+\sigma_{\text {inter }}(\omega)
$$

such that $\sigma_{\text {intra }}(\omega)=0$ at $\omega>\omega_{M}$ and $\sigma_{\text {inter }}(\omega)=0$ at $\omega<\omega_{M}$. As a consequence, the spectral weight $W\left(\omega_{M}, T\right)$ only measure $\sigma_{\text {intra }}(\omega)$ and gives a measure of the interactions acting on the system through the relation (2.13). When the integration frequency of Eq. (2.15) is extended above $\omega_{M}$ one should evolve towards the f-sum rule (2.14), and the inclusion of the processes described by $\sigma_{\text {inter }}(\omega)$ should give a temperature independent spectral weight. Observe that while many theoretical results have been provided in the literature about the behavior of the restricted spectral weight (2.13), based on different interacting model as Eq. (2.1), no clear theoretical understanding exists yet about the evolution of the spectral weight towards the f-sum rule as $\omega>\omega_{M}$ [14].

\section{B. Conventional spectral-weight behavior}

The relation between the optical-conductivity spectral weight and the diamagnetic tensor (or kinetic energy) introduced in the previous section is particularly useful from the theoretical point of view, because it allows one to derive the restricted sum rule without doing an explicit computation of the optical conductivity. However, one can also be interested on the precise structure of $\sigma(\omega)$, and on the eventual transfer of spectral weight through a phase transition. Before discussing the experimental data in the cuprates it is worth showing some examples of the applicability of the previous formulas in simple paradigmatic cases. 


\section{- Temperature dependence in the non-interacting nearest-neighbor tight-binding system}

In this case, in Eq. (2.13) the occupation number is simply given by the Fermi function, $n_{\mathbf{k} \sigma}=f\left(\xi_{\mathbf{k}}\right)$, where $\xi_{\mathbf{k}}=\varepsilon_{\mathbf{k}}-\mu$. The main temperature dependence of the spectral weight (2.13) comes from the temperature smearing of the Fermi function, and can be easily evaluated using the Sommerfeld expansion. In $n_{d}=2$ dimensions one obtains

$$
\frac{W(T)}{\left(\pi e^{2} a^{2} / V\right)}=-\frac{1}{N} \sum_{\mathbf{k}} \varepsilon_{\mathbf{k}} f\left(\xi_{\mathbf{k}}\right)=-\int d \varepsilon N(\varepsilon) \varepsilon f(\varepsilon-\mu)=\frac{W(0)}{\left(\pi e^{2} a^{2} / V\right)}-c(\mu) T^{2},
$$

where $c(\varepsilon)=\left(\pi^{2} / 6\right)\left[\varepsilon N^{\prime}(\varepsilon)+N(\varepsilon)\right]$, and $N(\varepsilon)$ and $N^{\prime}(\varepsilon)$ are the density of states (per spin) of the tight-binding dispersion and its derivative, respectively. For a flat density of states $N=1 / 2 D$ where $D$ is the semi-bandwidth, so that:

$$
\tilde{W}(T)=\tilde{W}(0)-\frac{\pi^{2}}{12 D} T^{2}=\tilde{W}(0)-B T^{2} .
$$

Observe that for a layered system $V=a^{2} d$, where $d$ is the inter-plane distance, so that the quantity $\tilde{W}=$ $W /\left(\pi e^{2} a^{2} / V\right)$ has the dimension of an energy (see discussion Sec. II-C below). The hopping $t$ of the twodimensional band dispersion can be related to the semi-bandwidth as $D=4 t$. Analogously, one can consider the tight-binding dispersion for $t^{\prime}=0$ and estimate, at small electron density, $N(\varepsilon)=1 / 4 \pi t$. The slope $c=\pi / 24 t$ so obtained is not far from the value of $c$ obtained around half filling, see Ref. [12].

\section{- Cut-off definition in the Drude model}

In metals displaying a conventional Fermi-liquid behavior the quasiparticles behave as free electrons with a renormalized mass $m^{*}$ and a characteristic scattering time $\tau=1 / \Gamma$. In the Drude approximation the real part of the optical conductivity reads:

$$
\operatorname{Re} \sigma_{\text {Drude }}(\omega, T)=\frac{n e^{2} \tau / m^{*}}{1+\omega^{2} \tau^{2}}=\frac{\Gamma}{\pi} \frac{W(T)}{\Gamma^{2}+\omega^{2}},
$$

where we made the dependence on the lattice spectral weight $W$ explicit. In Fig. 1 we report the integrated spectral weight as a function of the frequency. As one can see, at frequencies of the order of few times the inverse scattering time $\Gamma, 90 \%$ of the total value $W(T)$ is recovered, showing that in this case the cut-off frequency $\omega_{M}$ can be identified as $\omega_{M} \approx 6 \Gamma$ [4]. In a conventional metal one expects that Eq. (2.19) reproduces in good approximation the optical conductivity, eventually with a $\Gamma$ slightly temperature dependent. Moreover, the Fermi-liquid paradigm ensures that the quasiparticles still have a Fermi surface, with an occupation number $n_{\mathbf{k} \sigma}$ given by the Fermi function. The only effect of interactions is to renormalize the bandwidth, which can be for example determined with LDA calculations or extracted by some measurement of the Fermi surface (as for example by Angle Resolved Photoemission Spectroscopy (ARPES)). An example of such conventional Fermi-liquid behavior in gold has been indeed reported recently in Ref. [6].

To make contact with the notation that we will use below while discussing the experiments, we observe that for the Drude model (2.19) one usually expresses the spectral weight in terms of the energy $\Omega_{P}^{2}=4 \pi n e^{2} / m$, which corresponds to the relations:

$$
\sigma_{\text {Drude }}=\frac{\Omega_{P}^{2} / 4 \pi}{\Gamma-i \omega}, \quad W\left(\omega_{M}\right)=\frac{\Omega_{P}^{2}}{4} .
$$

Observe that in the Drude model the scale $\Omega_{p}$ is also related to the zero of the real part of the dielectric function $\varepsilon$, which is defined as:

$$
\varepsilon(\omega)=\varepsilon_{1}+i \varepsilon_{2}=\varepsilon_{\infty}+\frac{i 4 \pi \sigma(\omega)}{\omega},
$$

where $\varepsilon_{\infty}$ represents the screening by interband transitions. Using Eq. (2.20) we see that:

$$
\varepsilon_{1}^{\text {Drude }}(\omega)=\varepsilon_{\infty}-\frac{\Omega_{P}^{2}}{\Gamma^{2}+\omega^{2}} .
$$

As a consequence, we see that when $\Gamma \ll \Omega_{P}$ the real part of the dielectric function vanishes at a frequency $\omega_{0}$ given by:

$$
\varepsilon_{1}^{\text {Drude }}\left(\omega_{0}\right)=0 \rightarrow \omega_{0}^{2}=\frac{\Omega_{P}^{2}}{\varepsilon_{\infty}}\left(1-\frac{\Gamma^{2}}{\Omega_{P}^{2}}\right)
$$


so that within the Drude model $\omega_{0} \approx \Omega_{P} / \sqrt{\varepsilon_{\infty}}$ can also be related to the restricted spectral weight. Moreover, since in conventional metals $\Gamma \ll \Omega_{P}$, in these materials the term "plasma edge" refers both to the edge of intra-band optical absorption and to the spectral weight itself of the intra-band conductivity. However, the temperature dependence of $\omega_{0}$ is directly related to the temperature dependence of $\Omega_{P}$ (or $W$ ) only if $\Gamma$ is also temperature independent. Otherwise, a narrowing of the Drude peak with decreasing temperature can lead to a blue shift of $\omega_{0}$ which is not necessarily related to a change of the spectral weight $W$ [5].

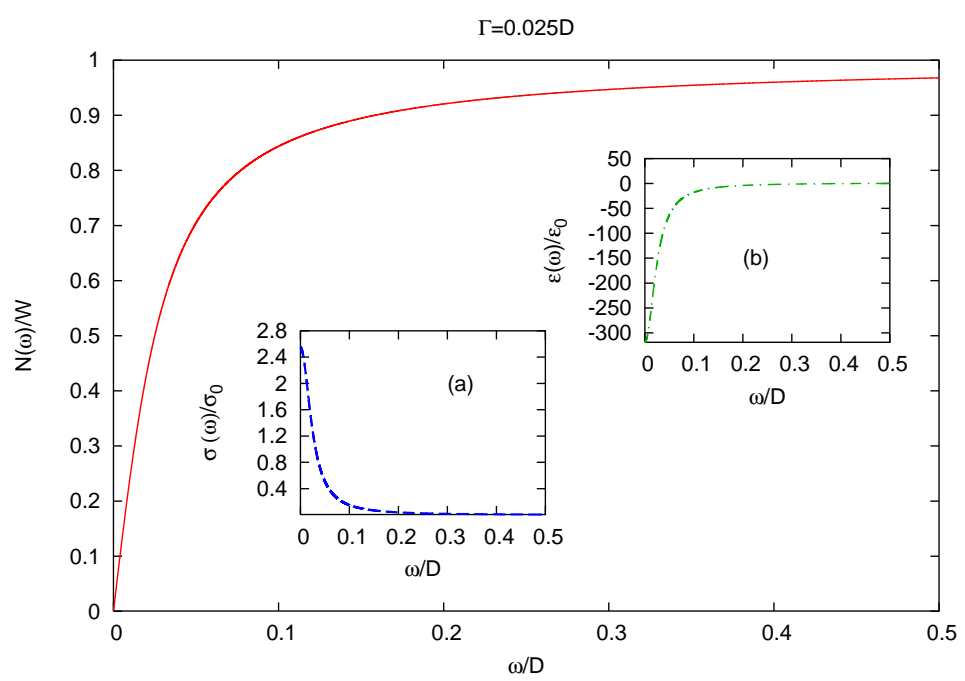

FIG. 1: Integral of the optical conductivity of the Drude model $N(\omega)=2 \int_{0}^{\omega} \operatorname{Re} \sigma_{D r u d e}\left(\omega^{\prime}\right) d \omega^{\prime}$, normalized to the total spectral weight $W$, as a function of $\omega / D$, where $D$ is the bandwidth. Inset (a); plot of the optical conductivity for $W=0.2 D$. Inset (b): real part of the dielectric function. Observe that here $\Omega_{P}=2 W=0.4 D$, where one can find the zero of the dielectric function.

\section{- Superconducting transition}

When a system displays a phase transition to a new ordered state, with CDW, SDW or SC formation, the occupation number $n_{\mathbf{k} \sigma}$ changes temperature dependence with respect to the simple Fermi function characteristic of the normal state of Fermi-liquid systems. A typical example is provided by the SC transition, where within the BCS approximation one can show that the spectral weight (2.11) acquires the form:

$$
W(\Delta, T)=\frac{2 \pi e^{2}}{V N} \sum_{\mathbf{k}} \frac{\partial^{2} \varepsilon_{\mathbf{k}}}{\partial k_{i}^{2}}\left[1-\frac{\xi_{\mathbf{k}}}{E_{\mathbf{k}}^{S C}} \tanh \frac{E_{\mathbf{k}}^{S C}}{2 T}\right]
$$

where $\Delta_{\mathbf{k}}=\left(\Delta_{0} / 2\right)\left(\cos k_{x} a-\cos k_{y} a\right)$ is the $d$-wave SC gap and $E_{\mathbf{k}}^{S C}=\sqrt{\xi_{\mathbf{k}}^{2}+\Delta_{\mathbf{k}}^{2}}$ is the quasiparticle dispersion in the SC state. One can easily see that the spectral weight $W(\Delta, T)$ decreases as $T$ is lowered below $T_{c}$. Bearing in mind Eq. (2.13), this decrease can be understood as a consequence of the kinetic-energy increase in the SC state, where the states above $\mu$ become occupied due to the particle-hole mixing.

\section{The experimental data in cuprates}

From the experimental point of view, the determination of the cut-off $\omega_{M}$ in Eq. (2.13) follows naturally from the observation of a minimum of the reflectivity (or of the conductivity) of cuprates at the so-called plasma edge $\omega_{P}$. This energy scale is of the order of $\omega_{P}=8000-10000 \mathrm{~cm}^{-1}$ in all the families of cuprates, and can be identified as the frequency $\omega_{0}$ at which the real part of the dielectric function crosses zero [6]. It is then usually assumed that transitions above $\omega_{P}$ involve interband processes, so that $\omega_{P}$ is a good candidate for the cut-off $\omega_{M}$ used in Eq. (2.13) to relate the spectral weight $W\left(\omega_{M}, T\right)$ to the diamagnetic tensor, according to Eq. (2.9). In this range one expects that the temperature dependence of $W(T)$ gives information about the interactions acting on the system.

While the identification between $\omega_{P}$ and $\omega_{M}$ is quite natural given the band structure of cuprates, the optical conductivity $\sigma(\omega)$ does not display at all the conventional Drude-like behavior represented by Eq. (2.19). A detailed discussion of all the peculiarities of the optical conductivity of cuprates is beyond the scope of this paper, and can be found for example in the recent reviews [19-21]. We only recall here that quite often the optical conductivity 
of cuprates is analyzed in terms of the so-called extended Drude model, which relies on an extension of Eq. (2.20) including a frequency-dependent optical mass enhancement $m^{*} \rightarrow m[1+\lambda(\omega)]$ and optical scattering time $\tau(\omega)$, according to the formula:

$$
\sigma(\omega)=\frac{\Omega_{P}^{2} / 4 \pi}{1 / \tau(\omega)-i \omega[1+\lambda(\omega)]}
$$

Although the representations of experimental data using $\operatorname{Re} \sigma(\omega), \operatorname{Im} \sigma(\omega)$ and $1 / \tau(\omega)$ with $1+\lambda(\omega)$ are formally equivalent, it has become rather popular to discuss the pseudogap behavior [19-21] in cuprate superconductors using the language of the optical scattering rate and mass enhancement. As we explained above, $\Omega_{P}$ is related the reduced spectral weight $W$. Indeed, normally $\Omega_{P}$ is found from the sum rule $(2.14)$ written in the form $\int_{0}^{\omega_{M}} \operatorname{Re} \sigma(\omega)=\Omega_{P}^{2} / 8$. As stressed in [20], the choice discussed here of the somehow arbitrary cut-off $\omega_{M}$ introduces an uncertainty in the overall scale factor of the optical scattering rate and optical mass in Eq. (2.25).

As one can see in Table I, $\Omega_{P}$ has values around $2 \mathrm{eV}$ in $\mathrm{Bi}_{2} \mathrm{Sr}_{2} \mathrm{CaCu}_{2} \mathrm{O}_{8}(\mathrm{Bi}-2212), \mathrm{Bi}_{2} \mathrm{Sr}_{2} \mathrm{Ca}_{2} \mathrm{Cu}_{3} \mathrm{O}_{10}(\mathrm{Bi}-2223)$ and $\mathrm{YBa}_{2} \mathrm{Cu}_{3} \mathrm{O}_{6+x}$ (YBCO) compounds, and somewhat smaller values in $\mathrm{La}_{2-x} \mathrm{Sr}_{x} \mathrm{CuO}_{4}$ (LSCO) samples. The analysis of the optical data is usually performed by using a cut-off $\omega_{M}$ temperature and doping independent, even though some variation in $\omega_{0}$ has been reported for example in BSCCO compounds [1]. However, as stressed in Ref. [5], the temperature variations of $\omega_{0}$ cannot be directly related to temperature variations of the spectral weight, because the temperature variations of $\omega_{0}$ are also affected by the thermal correction induced by the scattering time, see Eq. (2.23). In Table I we summarize some values of the low-temperature spectral weight taken from the experiments of Refs. [1-8]. As we explained before, the strength of the restricted partial weight $W$ can be expressed both as $W=\Omega_{P}^{2} / 4$ or in terms of the kinetic energy $K$ per $C u$ in-plane atom as in Eq. (2.13). To fix the energetic units, it can be useful to take into account the following relations. In most of the experiments the spectral weight $W_{\text {exp }}$ is defined as half of the value (2.13), because one integrates only the data at positive frequencies. Then from Eq. (2.13) in $n_{d}=2$ dimensions and (2.20) we have two relations:

$$
\Omega_{P}^{2}=4 W=8 W_{\exp }=E_{c} K,
$$

where the energy scale $E_{c}$ in appropriate units is:

$$
E_{c}=\frac{2 \pi e^{2} a^{2}}{V}=\frac{2 \pi e^{2}}{d}=\frac{2 \pi}{d} 14.38 \mathrm{eV} \stackrel{\AA}{ },
$$

and $d$ is the distance between $\mathrm{CuO}_{2}$ planes. To compare the data between single-layer and multi-layer compounds we used for $d$ the average distance between two neighboring layers, which corresponds approximately to $13.2 \AA, 6 \AA$, $7.5 \AA$ and $6.2 \AA$ in LSCO, YBCO, Bi-2212 and Bi-2223 compounds respectively. Since the spectral weight $W_{\exp }$ is measured usually in $\Omega^{-1} \mathrm{~cm}^{-2}$ one should also use the equivalence $1 \mathrm{~cm}^{-1}=0.21 \Omega^{-1} \mathrm{~cm}^{-1}$ [4]. Then one can obtain both the spectral weight in $\mathrm{cm}^{-2}$ (or equivalently in $\mathrm{eV}^{2}$ ), and the kinetic energy $K$, given by $8 W_{\text {exp }} / E_{c}=\Omega_{P}^{2} / E_{c}$. Finally, we note that the background dielectric constant $\varepsilon_{\infty}$ has typical values around 5 in cuprates, explaining the difference between $\omega_{P}$ and $\Omega_{P}$ in Table I.

\begin{tabular}{|c|c|c|c|c|c|}
\hline Ref. & Compound & $\omega_{M} \sim \omega_{P}(\mathrm{eV})$ & $\Omega_{P}(T=0)(\mathrm{eV})$ & $E_{c}(\mathrm{eV})$ & $K(T=0)(\mathrm{meV})$ \\
\hline$[1]$ & $\mathrm{Bi}-2212, T_{c}=66 \mathrm{~K}, \mathrm{UD}$ & & 1.90 & 12.04 & 300 \\
& $\mathrm{Bi}-2212, T_{c}=88 \mathrm{~K}$, OVD & 1.2 & 2.04 & & 346 \\
\hline$[2,3,7]$ & $\mathrm{Bi}-2212, T_{c}=70 \mathrm{~K}, \mathrm{UD}$ & 1 & 2.02 & 12.04 & 339 \\
& $\mathrm{Bi}-2212, T_{c}=80 \mathrm{~K}$, OPD & & 1.82 & & 275 \\
& $\mathrm{Bi}-2212, T_{c}=63 \mathrm{~K}, \mathrm{OVD}$ & & 2.12 & & 373 \\
\hline$[8]$ & $\mathrm{Bi}-2223, T_{c}=110 \mathrm{~K}$, OPD & 1 & 2.0 & 14.61 & 276 \\
\hline$[4]$ & YBCO, $T_{c}=57 \mathrm{~K}, \mathrm{UD}$ & 1 & 1.64 & 15.05 & 180 \\
& YBCO, $T_{c}=91 \mathrm{~K}$, OPD & 1 & 2.07 & & 285 \\
\hline$[5]$ & $\mathrm{YBCO}, T_{c}=92.7 \mathrm{~K}, \mathrm{OPD}$ & $0.5^{*}$ & 2.04 & 15.05 & 277 \\
\hline$[6]$ & $\mathrm{LSCO}, \mathrm{x}=012, \mathrm{UD}$ & 0.8 & 1.41 & 6.84 & 290 \\
& $\mathrm{LSCO}, \mathrm{x}=0.26$, OVD & & 1.78 & & 463 \\
\hline
\end{tabular}

Table I: Low-temperature spectral weight in several families of cuprates and several doping (UD=underdoped, $\mathrm{OPD}=$ optimally doped, $\mathrm{OVD}=$ ovedoped). The data at $T=0$ are either extrapolated from the data at $T>T_{c}$, 
as in Ref. $[1-3,6-8]$, or are taken at $T \approx T_{c}$, as in $[4,5]$. Observe that the absolute value of the spectral weight determined in Ref. [2,3] is affected by an error of 15-20\%, which justifies the fact that $\Omega_{P}$ it is lower in the OPD sample than in the UD one. * (The data for YBCO extracted from Ref. [5] refer to the integral of the spectral weight up to $0.5 \mathrm{eV}$. However, the plasma edge reported in this article is still of order of $1 \mathrm{eV}$.)

In Table I we reported the values of the spectral weight measured at low temperature as a function of doping. The first observation is that the partial spectral weight increases when the doping increases. This behavior is in agreement with the general observation that cuprate superconductors are doped Mott insulators [22]. Indeed, these systems behave as if the effective number of charge carriers is proportional to the doped holes instead of the total number of electrons, so that the spectral weight increases when the system is doped with respect to half filling. It is worth noting that this same behavior was observed long ago for the doping dependence of the superfluid density [23], which is also proportional to the partial spectral weight, as we shall discuss below.

While the doping dependence of the $T=0$ spectral weight is in some sense expected for a doped Mott insulator, the recent experimental data focused on some new interesting aspects related to the temperature dependence of $W(T)$, both in the normal and in the superconducting state. As far as the range of temperature $T>T_{c}$ is concerned, three main observations have been made: (i) in all the compounds analyzed in Ref. [1-6] $W\left(\omega_{P}, T\right)$ shows a $T^{2}$ decreasing as the temperature increases, but with a coefficient $B\left(\omega_{P}\right)$ much larger than the one derived in Eq. (2.18) for a standard Fermi liquid; (ii) when the cut-off frequency $\omega_{M}$ is lower than the plasma edge $\omega_{P}$ the coefficient $B\left(\omega_{M}\right)$ becomes even larger; (iii) in underdoped compounds the opening of the pseudogap does not affect the restricted partial weight, in particular it does not lead to any suppression of the spectral weight, as one could expect as a consequence of a (pseudo)gap opening.

This last observation is intimately related to the typical behavior of the spectral weight below the SC transition, as described by the BCS relation (2.24). Indeed, if one assumes that the pseudogap opening is due to some form a preformed Cooper pairing, then one could also expect to see some decrease of the spectral weight, due to the same mechanism which leads to the decrease of $W$ across the BCS transition. The intriguing question arises how to reconcile the opening of the the pseudogap with an almost conventional temperature dependence of the spectral weight, where, however, the effect of the correlations appears in a renormalization of the coefficient $B$ of Eq. (2.18). To be more specific, one can extract from the experiments the coefficient $B_{\text {exp }}$ as:

$$
W_{\text {exp }}(\Omega, T)=W_{\text {exp }}(\Omega, 0)-B_{\text {exp }}(\Omega) T^{2} .
$$

According to the relations (2.18) and (2.26) for $\Omega=\omega_{M}=\omega_{P}$ one can relate $B_{\exp }$ to the value $B=\pi^{2} / 12 D$ of Eq. (2.18) obtained within a tight-binding model:

$$
B_{\text {exp }}=\frac{\pi e^{2}}{d} \frac{\pi^{2}}{24 D}=\frac{E_{c} B}{4} .
$$

We can then extract from $B_{\text {exp }}$ an effective hopping parameter $t_{T}=\pi^{2} / 48 B$ corresponding to the thermal variation of the spectral weight. As one can see in Table II, the $t_{T}$ values extracted in this way are significantly smaller than the ones obtained by ARPES measurements of the Fermi surface of cuprates, which are usually of the order of $t \sim 300$ meV, leading to a temperature variation $B \sim 1 / t_{T}$ larger than expected in a standard Fermi liquid. Even in samples were the quadratic temperature dependence has not been clearly observed, the large temperature variation is made apparent by analyzing the relative spectral weight variations $\Delta W / W=[W(0)-W(300 K)] / W(0)$, reported in the last column of Table II. Observe indeed that in the usual (non-interacting) tight-binding model $\Delta W / W$ never exceeds the value of about $0.5 \%$ [12]. It is worth noting that in the first works of Molegraaf et al. [1] and Santander-Syro et al. [2] on the spectral-weight behavior on cuprates the attention was focused mainly on the absence of spectral-weight decrease below the pseudogap temperature, but not on the anomalously large values of $B$ measured in these BSCCO samples. This issue was addressed for the first time from the theoretical point of view in Ref. [11]. More recently Ortolani et al. [6] clearly stated this problem in their analysis of the optical data of LSCO compounds. As we shall discuss in the following Sections, motivated by the observation of the anomalous large value of $B$ we investigated indeed the possibility to reconcile the large slope of $W(T)$ with a DDW model for the pseudogap phase of cuprates. 


\begin{tabular}{|c|c|c|c|c|}
\hline Ref. & Compound & $B\left(\mathrm{eV}^{-1}\right)$ & $t_{T}(\mathrm{meV})$ & $\Delta W / W(0)$ \\
\hline$[1]$ & $\mathrm{Bi}-2212, T_{c}=66 \mathrm{~K}, \mathrm{UD}$ & 9.3 & 21 & 0.021 \\
& $\mathrm{Bi}-2212, T_{c}=88 \mathrm{~K}$, OVD & 11.3 & 18 & 0.022 \\
\hline$[2,3,7]$ & $\mathrm{Bi}-2212, T_{c}=70 \mathrm{~K}, \mathrm{UD}$ & 10.8 & 18 & 0.042 \\
& $\mathrm{Bi}-2212, T_{c}=80 \mathrm{~K}, \mathrm{OPD}$ & 10.8 & 18 & 0.053 \\
& $\mathrm{Bi}-2212, T_{c}=63 \mathrm{~K}, \mathrm{OVD}$ & 16.4 & 12 & 0.053 \\
\hline$[8]$ & $\mathrm{Bi}-2223, T_{c}=110 \mathrm{~K}, \mathrm{OPD}$ & 4.2 & 47 & 0.018 \\
\hline$[6]$ & $\mathrm{LSCO}, \mathrm{x}=012$ & 8.3 & 24 & 0.042 \\
& $\mathrm{LSCO}, \mathrm{x}=0.26$ & 10.4 & 19 & 0.026 \\
\hline
\end{tabular}

Table II: Slope $B$ of the $T^{2}$ temperature variation of the spectral weight in several families of cuprates. We report here the value obtained integrating the spectral weight up to the cut-off $\omega_{M}=\omega_{P}$ as reported in Table I for the same compounds. The 'thermal' estimate of the hopping $t_{T}$ is evaluated using a flat band dispersion, see discussion below Eq. (2.29). Last column: relative variation of the spectral weight between $T=0$ and $T=300 \mathrm{~K}, \Delta W / W=$ $[W(0)-W(300 K)] / W(0)$.

Finally, we describe the evolution of spectral weight in the superconducting state. When the SC order is established, part of the low-frequency spectral weight moves into the SC response, represented by a singular contribution to the optical conductivity:

$$
\sigma_{\text {singular }}^{S C}(\omega)=\frac{e^{2} n_{s}}{m}\left[\pi \delta(\omega)+\frac{\mathrm{i}}{\omega}\right]
$$

where $n_{s}$ is the superfluid density, related to the penetration depth $\lambda$ by the usual relation $1 / \lambda^{2}=4 \pi e^{2} n_{s} / m$. As a consequence, below $T_{c}$ the real part of the conductivity reads:

$$
\operatorname{Re} \sigma(\omega)=\frac{\pi e^{2} n_{s}}{m} \delta(\omega)+\sigma_{r e g}
$$

where $\sigma_{\text {reg }}$ is the regular part of the conductivity below $T_{c}$. As far as the sum rule (2.9) is concerned, one sees that the condensate contribution is always included in $W$, and the effect of the SC transition is already taken into account by the general formula (2.13) through the change in the occupation number. For example, in the BCS theory the spectral weight is expected to decrease below $T_{c}$, see Eq. (2.24). However, from the experimental point of view the definition (2.31) is useful to correctly determine the spectral weight $W$ by taking into account also the condensate contribution. Thus, one usually integrates the spectral weight of the regular part up to $\omega_{s c}, W_{r e g}\left(\omega_{s c}\right)=\int_{0^{+}}^{\omega_{s c}} \operatorname{Re} \sigma_{r e g}(\Omega) d \omega$, obtained from the finite-frequency measurement of the conductivity, and estimate the superfluid weight as $W_{s}=\pi e^{2} n_{s} / m$, usually using the slope of the inverse imaginary part of $\sigma_{\text {singular }}^{S C}$, see Eq. (2.30). Then the value $W=W_{s}+W_{\text {reg }}\left(\omega_{s c}\right)$ can be compared with the theoretical prediction, which usually concerns the spectral weight integrated up to the cut-off $\omega_{s c}=\omega_{M}$. In particular, the BCS model (2.24) predicts a decrease of $W$ as $T$ decrease in the SC state, to be contrasted to the increase expected in the normal state according to Eq. (2.18). Even though all the procedure described above can be affected by a significant error, due mainly to the exact determination of the superfluid weight, nonetheless it was reported by two different group that in an underdoped and an optimally-doped Bi-2212 compound [1] and in an underdoped Bi-2212 film [2] there is an effective increase of the spectral weight in the SC state with respect to the value in the normal state. More specifically, it was shown that by integrating $W_{\text {reg }}$ up to the cut-off $\omega_{M} \sim \omega_{P} W(T)$ is still increasing as $T$ decreases, with a temperature variation even larger than the value $B$ found in the normal state, see Eq. (2.28). This result has been recently confirmed in a slightly underdoped Bi-2223 sample [8]. Later on, Santander et al. showed that in the optimally-doped sample the spectral weight is almost constant in the SC state, with $W\left(T<T_{c}\right) \approx W\left(T_{c}\right)$ [3], as it seems to be the case also for LSCO [6]. Finally, a more conventional result has been observed in an overdoped Bi-2212 film [7], showing that the anomalous spectral-weight behavior can be eventually doping dependent. However, these results has been questioned in Ref. [5], where the optical data of an optimally-doped YBCO sample and slightly-underdoped Bi-2212 sample up to a larger cut-off of $\sim 1.5 \mathrm{eV}$ were considered. According to the analysis of Boris et al., the spectral weight of YBCO and Bi-2212 in this frequency range keeps constant in the normal state and decrease when the SC state is formed. Thus, the issue of the spectral-weight behavior below $T_{c}$ remains at the moment quite controversial $[9,10]$, and seems to be related mainly to the analysis of the optical data rather than to an effective discrepancy between them. In particular, it seems to us that in Ref. [5] it is mainly questioned the choice of the plasma edge $\omega_{P} \sim 1 \mathrm{eV}$ as the proper cut-off $\omega_{M}$ for the analysis of optical data. However, this is the most plausible choice from the theoretical point of view, because extending the spectral-weight 
integration to interband processes one naturally recovers the full f-sum rule (2.14), which is just a constant and does not provide any significant information on the interactions acting on the system.

\section{OPTICAL CONDUCTIVITY SUM RULE FOR A REDUCED $d$-DENSITY WAVE MODEL}

While it is clear that any successful theory of HTSC should be able to explain the above-mentioned experimental facts, despite the 20 year jubilee since the discovery of HTSC, there is no consensus yet neither on the general theory of HTSC, nor on the particular explanation of these facts.

Although our goal is to present here the latest theoretical results for the optical-conductivity sum rule within the d-density wave paradigm, we would like to mention some other works related to this issue in the recent literature. A first class of papers is related to the spectral-weight below the superconducting critical temperature. For example, the possibility of a spectral-weight change below $T_{c}$ in terms of the lowering of the in-plane kinetic energy has been analyzed in Refs. [24-27]. In Ref. [24] a model with "occupation modulated" hopping terms was considered. The reduction of the kinetic energy at $T_{c}$ was attributed in Refs. $\left.[26,27]\right)$ to the transition from a phase-incoherent Cooper pair motion in the pseudogap regime above $T_{c}$ (see, e.g. review [28]) to a phase coherent motion at $T_{c}$, while in Ref. [25] a model with a frequency dependent scattering rate was used. More recently, the optical-conductivity sum rule was analyzed for a model with electrons in the finite band coupled to a single Einstein oscillator [29] and in the nearly antiferromagnetic Fermi liquid model [30].

As far as the anomalous behavior of the spectral weight in the normal-state is concerned, a possible interpretation was recently given by Toschi et al. [22]. In this work the authors evaluate the spectral-weight behavior within the dynamical mean-field analysis of the repulsive Hubbard model, and they show that the large slope $B$ (see Eqs. (2.18) and (2.28)) is a consequence of the bare-bandwidth renormalization $B \sim 1 / Z t$ where $Z \rightarrow 0$ as the Mott-insulator is approached. At the same time the $T=0$ value of $W$ is much less affected by the $Z$ renormalization, due to the contribution of the so-called mid-infrared processes, which occur below $\omega_{P}$ and partly compensate for the reduction of the quasi-particle weight [22].

Finally, the behavior of the spectral weight in the DDW state was addressed specifically in a series of recent papers, Ref. [11-13, 31, 32]. While the issue of the vertex corrections, discussed below, is the most important ingredient of the Refs. [11-13], in Ref. [31] they were ignored and the main attention was paid to the analysis of the frequency dependence of $\sigma(\omega)$ both in the presence and in the absence of the next-nearest-neighbor hopping. As we will see in Sec. IV, the results of Ref. [31] are particularly useful for the discussion of interband optical transitions when the vertex correction becomes unimportant. At the same time, Ref. [32] investigates the consequences of a momentum-dependent scattering time on the optical spectra, neglecting again the contribution of vertex corrections.

\section{A. Unconventional charge density waves}

Since 2000 the most commonly used name for the state that we consider in the present article is d-density wave $(D D W)$ state [33]. It is considered as one among competing theoretical approaches that are attempting to explain the pseudogap behavior in cuprates [21]. However, we do not intend to analyze here to which extent the DDW model can explain the vast body of the experimental data accumulated during the last 20 years. Instead of this we will concentrate on rather important theoretical subtleties of the DDW model that are often overlooked and their implications for the optical conductivity. Yet before writing down the model Hamiltonian we find useful to remind the reader of the rather long history of the model.

The DDW or, as it was called originally, the orbital antiferromagnetic state was introduced in solid state physics in Ref. [34]. The discovery of HTSC and searches for a model that would describe the $\mathrm{CuO}_{2}$ planes without phonons lead to the investigation of the Heisenberg-Hubbard model [35-38] and to the rediscovery of this state which at this time was called a staggered flux phase. The phase with circulating orbital currents turned out to be an attractive idea and there are hundreds of papers (see, for instance, very few of them in Refs. [39-43]) that exploit and develop it. These studies showed that the DDW state can be obtained when the interaction term in (2.1), for example, is Heisenberg

$$
H_{i n t}=\frac{J}{2} \sum_{\substack{<i j>\\ \alpha \beta \gamma \delta}} c_{i \alpha}^{\dagger} \vec{\sigma}_{\alpha \beta} c_{i \alpha} c_{j \gamma}^{\dagger} \vec{\sigma}_{\gamma \delta} c_{j \delta},
$$

or Hubbard-like

$$
H_{i n t}=\sum_{i j, \sigma \sigma^{\prime}} c_{i \sigma}^{\dagger} c_{i \sigma} V\left(\mathbf{r}_{i}-\mathbf{r}_{j}\right) c_{j \sigma^{\prime}}^{\dagger} c_{j \sigma^{\prime}}
$$


The sum in Eq. (3.1) runs over nearest neighbors sites $i$ and $j, \vec{\sigma}$ is the vector of Pauli matrices and $V\left(\mathbf{r}_{i}-\mathbf{r}_{j}\right)$ in Eq. (3.2) is a generic density-density interaction. For example, the repulsive Hubbard model corresponds to the case $V\left(\mathbf{r}_{i}-\mathbf{r}_{j}\right)=U$ for $i=j$.

In the mean-field treatment the expectation value of any two operators, i.e. the order parameter, may be nonzero. In particular, if the expectation value of two operators with the same spin projection is nonzero we deal with a charge-density wave (CDW), which can have an internal symmetry if the two operators in the order parameter belong to different sites. CDW with an internal symmetry are called unconventional charge-density waves to distinguish them from conventional ones, where the electron and hole sit on the same site. An other possibility is that the order parameter describes microscopic currents running on few sites of the lattice, as it is the case for the flux phase [35-38] or for the current patterns proposed by Varma [39]. It is worth noting that strictly speaking the flux phase does not present a charge modulation, even though a gap is present in the excitation spectrum. Indeed, the same phenomenological spectrum of the DDW state has been proposed as emerging in cuprates due to the tendency of the system to form charge order. This attitude was for example considered in Ref. [42], where the DDW state emerges as the consequence of the proximity of the system to a quantum critical point [39, 44]. More generally, unconventional charge density waves have been observed in many other systems, as for example organic conductors [43] or dichalcogenides [45, 46], but we will restrict our discussion here to cuprate superconductors. In the following discussion it will be clear that the crucial point in the evaluation of the optical conductivity for a state with DDW or, more general, with a CDW with internal symmetry, is the non-trivial dependence of the electronic self-energy on the momentum. For this reason the results reported below are generic to many CDW systems, even though we will consider specifically the case of DDW order in cuprate superconductors.

\section{B. Reduced $d$-density wave model}

In the DDW case, the general interaction term (3.2) is replaced by an approximate reduced interaction Hamiltonian:

$$
H_{I}=-\frac{V_{0}}{2 N} \sum_{\substack{k, k^{\prime} \\ \sigma, \sigma^{\prime}}} w_{d}(\mathbf{k}) w_{d}\left(\mathbf{k}^{\prime}\right) c_{\mathbf{k} \sigma}^{\dagger} c_{\mathbf{k}+\mathbf{Q} \sigma} c_{\mathbf{k}^{\prime}+\mathbf{Q} \sigma^{\prime}}^{\dagger} c_{\mathbf{k}^{\prime} \sigma^{\prime}},
$$

where $w_{d}(\mathbf{k})=\left(\cos k_{x} a-\cos k_{y} a\right) / 2$ corresponds to $d$-wave symmetry with respect to the discrete rotation group of the square lattice. By defining $i D_{0}=-\left(V_{0} / N\right) \sum_{\mathbf{k} \sigma} w_{d}(\mathbf{k})<c_{\mathbf{k}+\mathbf{Q} \sigma}^{\dagger} c_{\mathbf{k} \sigma}>$ and adding the non-interacting Hamiltonian $H_{0}$ from (2.1) we arrive at the mean-field DDW Hamiltonian

$$
H=\sum_{\mathbf{k}, \sigma}\left[\left(\varepsilon_{\mathbf{k}}-\mu\right) c_{\mathbf{k} \sigma}^{\dagger} c_{\mathbf{k} \sigma}+i D_{\mathbf{k}} c_{\mathbf{k} \sigma}^{\dagger} c_{\mathbf{k}+\mathbf{Q} \sigma}\right]
$$

where $D_{\mathbf{k}}=D_{0} w_{d}(\mathbf{k})$ is the gap, known as the DDW gap [33], arising from the formation of the state with circulating currents below a characteristic temperature $T_{D D W}$. In writing Eq. (3.4) we considered the case of $t^{\prime}=0$ in the bare band dispersion, so that the nesting condition $\varepsilon_{\mathbf{k}+\mathbf{Q}}=-\varepsilon_{\mathbf{k}}$ is satisfied, where $\mathbf{Q}=(\pi / a, \pi / a)$ is the characteristic wave-vector at which a particle-hole coupling in the DDW state is considered. However, the following results are easily extended to the case $t^{\prime} \neq 0$ (see, for example, Refs. [12, 31, 47]).

The notation is then simplified by halving the Brillouin zone and introducing two-component electron operators (the DDW equivalent of Nambu spinors [48] widely used in the theory of superconductivity [17])

$$
\chi_{\mathbf{k} \sigma}^{\dagger}=\left(\begin{array}{cc}
c_{\mathbf{k} \sigma}^{\dagger} & c_{\mathbf{k}+\mathbf{Q}, \sigma}^{\dagger}
\end{array}\right)
$$

where $c_{\mathbf{k} \sigma}^{\dagger}$ and $c_{\mathbf{k} \sigma}$ are the Fourier transforms of $c_{i \sigma}^{\dagger}$ and $c_{i \sigma}$. The Hamiltonian (3.4) written in terms of $\chi$ becomes

$$
H=\sum_{\mathbf{k}, \sigma}^{R B Z} \chi_{\mathbf{k} \sigma}^{\dagger}\left[\varepsilon_{\mathbf{k}} \sigma_{3}-D_{\mathbf{k}} \sigma_{2}-\mu\right] \chi_{\mathbf{k} \sigma},
$$

where the sum is taken over the reduced (magnetic) Brillouin zone (RBZ). Thus one can easily see that when $D_{0} \neq 0$ two quasiparticles excitation branches are formed with dispersion $\xi_{ \pm, \mathbf{k}}=-\mu \pm E_{\mathbf{k}}$, where $E_{\mathbf{k}}=\sqrt{\varepsilon_{\mathbf{k}}^{2}+D_{\mathbf{k}}^{2}}$. As a consequence, at finite doping with respect to half-filling the Fermi surface consists of small pockets around the $( \pm \pi / 2, \pm \pi / 2)$ points and a finite leading-edge shift $L E \approx D_{0}-\mu$ at the $M$ points, which mimic in some sense the arc of Fermi surface observed by ARPES measurements in the underdoped phase of cuprates [31, 42]. For this reason, 
the Hamiltonian (3.4) can be considered as an "effective" model for the normal (i.e. non SC) pseudogap phase of cuprates, where SC forms within a standard BCS mechanism, since all the anomalies related to the presence of a pseudogap are already included in the Hamiltonian (3.4). This point of view was adopted for example in Ref. [42], where the phase diagram of Fig. 2 was derived, in excellent agreement with the experimental data on BSCCO.
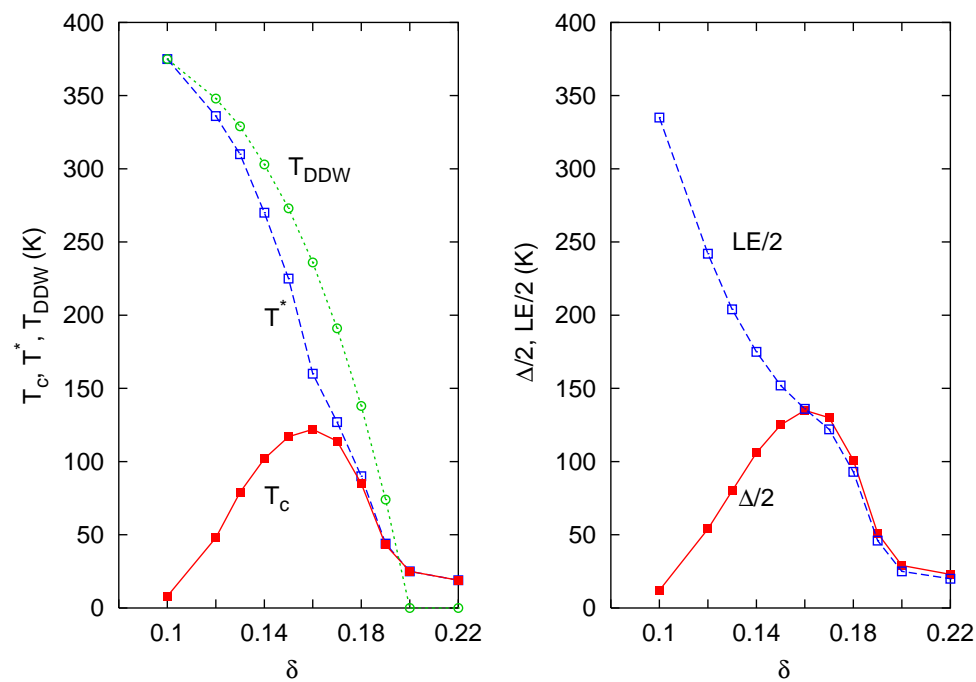

FIG. 2: Phase diagram of cuprates [taken from Ref. [42]] derived adding the BCS pairing term (3.12) to the Hamiltonian (3.4). $\quad T_{D D W}$ is the temperatures where $D_{0}$ forms, $T^{*}$ is the temperature where a leading-edge (LE) shift appears in the quasiparticle spectrum at the $M$ points, and $T_{c}$ is the SC temperature, at which the SC gap $\Delta$ forms.

If one consider the Hamiltonian (3.4) as an effective model, it is straightforward to calculate the current-current correlation function and the electrical conductivity for it, because it describes non-interacting quasiparticles. Nevertheless, one should be careful and take into account that the current operator and the diamagnetic tensor for the Hamiltonian (3.4) are different from those given by Eqs. (2.10) and (2.11) for the Hamiltonian (2.1).

This can be understood by deriving the particle current operator compatible with the charge conservation law and with the equations of motion for the operators $c$ and $c^{\dagger},[47,49-51]$

$$
\mathbf{j}(\mathbf{q}, t)=\frac{1}{N} \sum_{\mathbf{k}, \sigma}\left[v_{\mathbf{k}}^{F} c_{\mathbf{k}-\mathbf{q} / 2 \sigma}^{\dagger} c_{\mathbf{k}+\mathbf{q} / 2 \sigma}-i v_{\mathbf{k}}^{D} c_{\mathbf{k}-\mathbf{q} / 2 \sigma}^{\dagger} c_{\mathbf{k}+\mathbf{Q}+\mathbf{q} / 2 \sigma}\right]
$$

where $v_{\mathbf{k}_{i}}^{D}=-\partial D_{\mathbf{k}} / \partial \mathbf{k}_{i}$ is the DDW gap velocity. The first term of the previous expression relates as usual the particle current to the band velocity $v_{\mathbf{k}}^{F}$, see Eq. (2.12). The second term, which only appears for non-vanishing $D_{0}$, takes into account the contribution of the orbital currents to the electrical conductivity, and it emerges when the DDW order develops. Observe that in the reduced model (3.4) the term proportional to $D_{0}$ appears as an additional, temperature dependent band, which couples $\mathbf{k}$ and $\mathbf{k}+\mathbf{Q}$ electrons, and as a consequence a corresponding term appears in the definition of the current. By rewriting the electric current operator (3.7) using the spinors (3.5), one has

$$
j_{i}(\mathbf{0}, t)=\frac{1}{N} \sum_{\mathbf{k} \sigma}^{\mathrm{RBZ}} \chi_{\mathbf{k} \sigma}^{\dagger} V_{i}(\mathbf{k}) \chi_{\mathbf{k} \sigma}
$$

where $V_{i}(\mathbf{k})$ is a generalized velocity which extends the definition (2.12) to the case of Hamiltonian (3.4)

$$
V_{i}(\mathbf{k}) \equiv v_{\mathbf{k}_{i}}^{F} \sigma_{3}+v_{\mathbf{k}_{i}}^{D} \sigma_{2}
$$

Note that this result appears to be rather trivial, if one considers the original paper [35], where the staggered flux phase was introduced, and the sum of hopping and staggered flux terms was considered as the "effective" hopping term.

The modification induced by the DDW order parameter on the current operator demands a consistent change in the diamagnetic tensor with respect to the definition (2.11). As we discussed in Sec. II A, after the Peierls substitution both the current operator and the diamagnetic tensor can be derived from $H(A)$, according to Eq. (2.2). As a 
consequence, by performing the Peierls substitution in the reduced model (3.4) not only the current operator but also the diamagnetic tensor $\left\langle\tau_{i i}\right\rangle$ is modified, containing an extra term for $D_{0} \neq 0$ [11],

$$
\left\langle\tau_{i i}\right\rangle=-\frac{a^{2}}{2 N} \sum_{\mathbf{k} \sigma}\left[\varepsilon_{\mathbf{k}}\left\langle c_{\mathbf{k} \sigma}^{\dagger} c_{\mathbf{k} \sigma}\right\rangle+i D_{\mathbf{k}}\left\langle c_{\mathbf{k} \sigma}^{\dagger} c_{\mathbf{k}+\mathbf{Q} \sigma}\right\rangle\right] .
$$

When the operator averages are evaluated (see Refs. $[11,12]$ for the details), one finds that the sum rule for the reduced model is:

$$
\frac{W^{D D W}(D, T)}{\left(\pi e^{2} a^{2} / V\right)}=-\frac{1}{N} \sum_{\mathbf{k}}^{R B Z} E_{\mathbf{k}}\left[f\left(\xi_{+, \mathbf{k}}\right)-f\left(\xi_{-, \mathbf{k}}\right)\right] .
$$

Eq. (3.11) was derived using the fact that $\partial_{x, y} v_{\mathbf{k}}^{F}=2 t a^{2} \cos k_{x, y} a$ (and $\partial_{x, y} v_{\mathbf{k}}^{D}= \pm\left(D_{0} / 2\right) a^{2} \cos k_{x, y} a$ ), and it reduces to Eq. (2.13) for $D_{0}=0$. Another way to derive the result (3.11) is to evaluate the commutator of the density $j_{0}(\mathbf{q}, t)$ and the current $j_{i}(\mathbf{q}, t)$, given by Eq. (3.7), as explained in Refs. [11, 12].

As we mentioned above, one can consider the superconductivity formation on top of the DDW state as arising from a conventional BCS type of pairing. More specifically, we can add to the Hamiltonian (3.6) an additional d-wave mean-field pairing term

$$
H_{p}=\sum_{\mathbf{k}}\left[\Delta_{\mathbf{k}}^{*} c_{-\mathbf{k} \downarrow} c_{\mathbf{k} \uparrow}+\text { h.c. }\right]
$$

where the SC $d$-wave gap $\Delta_{\mathbf{k}}$ is defined after Eq. (2.24). In the SC phase the occupation numbers of Eq. (3.10) get modified, and the spectral weight in the DDW+SC state reads

$$
\frac{W^{D D W}(D, \Delta, T)}{\pi e^{2} a^{2}}=\frac{1}{2 V N} \sum_{\mathrm{RBZ}} E\left[\frac{\xi_{+}}{E_{+}} \tanh \frac{E_{+}}{2 T}-\frac{\xi_{-}}{E_{-}} \tanh \frac{E_{-}}{2 T}\right],
$$

where $E_{ \pm, \mathbf{k}}=\sqrt{\xi_{ \pm, \mathbf{k}}^{2}+\Delta_{\mathbf{k}}^{2}}$ is the quasiparticle dispersion in the presence of pairing, and the explicit dependence on $\mathbf{k}$ has been omitted.

\section{Results for the reduced model}

The numerical calculation of the spectral weight defined by Eqs. (3.11) and (3.13) was done in Ref. [11] and the results at doping $\delta=0.13$ are reported in Fig. 3. As one can see, below $T_{D D W}$ the spectral weight $W^{D D W}$ defined in Eq. (3.11) is larger than the value obtained in the absence of the DDW order, represented by the dotted-dashed line in Fig. 3. When also SC is established, the spectral weight $W^{D D W}(D, \Delta)$ given by Eq. (3.13) is slightly smaller than in the DDW state only, the change being proportional to the ratio $\Delta / D_{0}$ which is quite small at this doping. In the inset of Fig. 3 we plot $W(T)$ as a function of $T^{2}$. One can see that below $T_{D D W}$ the $T^{2}$ temperature dependence of $W(T)$ is still recovered over a wide range of temperature, but with a slope much larger than in the non-DDW case. All these results show some interesting resemblance to the experimental data for cuprates discussed in Sec. II-C. In particular, it turns out that the large spectral-weight variation measured by the experiments of Ref. $[1-3,6-8]$ between $T=0$ and $T=300 \mathrm{~K} \sim 0.1 t$ can be ascribed to existence of DDW order in the normal state. Moreover, when SC is formed on top of this DDW state the spectral weight remains almost constant, as observed in Ref. [3]. However, one should notice that on the overdoped side, the temperature $T_{D D W}$ of DDW formation decreases (see Fig. 2) so that $T_{D D W}$ can be smaller than room temperature, and a change of slope of the spectral weight at $T_{D D W}$ should be observed. Up to now, no signature of this effect has been observed in the experiments.

To develop a better insight into the origin of the spectral-weight increase in the DDW state we calculated in Ref. [12] the optical conductivity corresponding to the sum rule (3.11). This can be done using the general definition (2.6) where, however, the correlation function (2.5) is modified according to the new current definition (3.7). Let us first introduce the Green's function (GF) corresponding to the effective Hamiltonian (3.6):

$$
G^{-1}\left(\mathbf{k}, i \omega_{n}\right)=\left(i \omega_{n}+\mu\right) \sigma_{0}-\varepsilon_{\mathbf{k}} \sigma_{3}+D_{\mathbf{k}} \sigma_{2} .
$$

Using this GF one can write down an exact expression for the correlation function

$$
\Pi_{i j}\left(\mathbf{q}=\mathbf{0}, i \Omega_{m}\right)=-2 \frac{T}{N} \sum_{\mathbf{k}, i \omega_{n}}^{R B Z} \operatorname{Tr}\left[G\left(\mathbf{k}, i \omega_{n}+i \Omega_{m}\right) \gamma_{i}(\mathbf{k}) G\left(\mathbf{k}, i \omega_{n}\right) \gamma_{j}(\mathbf{k})\right],
$$




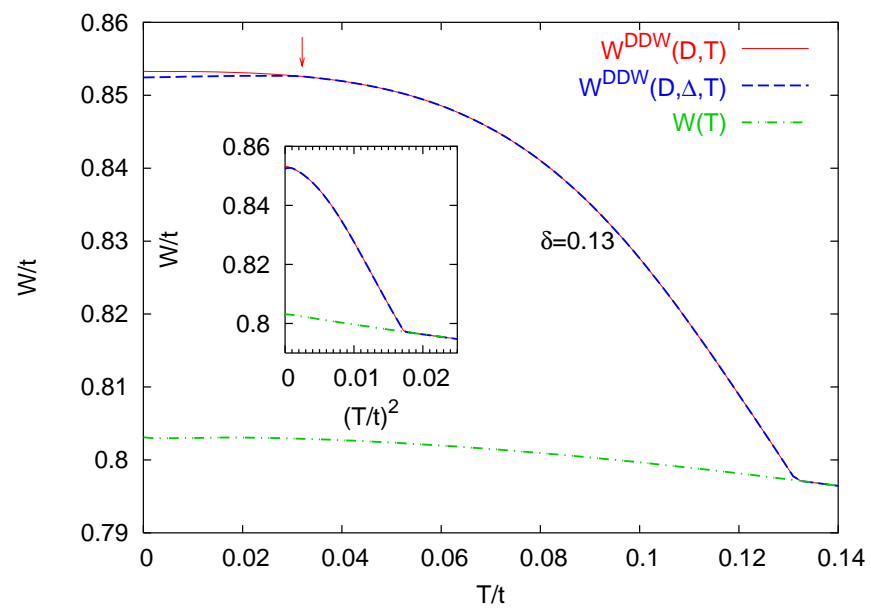

FIG. 3: Spectral weight in units of $e^{2} \pi a^{2} / V$ in the normal state Eq. (2.17) (dot-dashed line), in the DDW state Eq. (3.11) (dashed line) and in DDW+SC state Eq. (3.13) (full line). The values of parameters are the same used in Fig. 2 for the doping $\delta=0.13$ $\left(D_{0}(T=0)=0.92 t, \Delta(T=0)=0.064 t\right.$, see Ref. [11, 42] for further details). The critical temperature is marked by the arrow. Observe that the decrease of $W^{D D W}(D, \Delta, T)$ below $T_{c}$ is almost negligible. Inset: spectral weight plotted as function of $(T / t)^{2}$.

where

$$
\gamma_{i}(\mathbf{k})=V_{i}(\mathbf{k})
$$

with $V_{i}(\mathbf{k})$ defined in Eq. (3.9) is the bare vertex for our noninteracting theory [52]. One can also convince oneself that (3.16) is indeed the appropriate vertex for the reduced Hamiltonian (3.4) by looking at the Ward identity (see Eq. (4.4) below)

$$
\gamma_{i}(\mathbf{k})=-\frac{\partial G^{-1}\left(\mathbf{k}, i \omega_{n}\right)}{\partial \mathbf{k}_{i}}
$$

which is just the charge-conservation law expressed in terms of the Green's and vertex functions. Then one can state that the term $\sim \sigma_{2} v_{\mathbf{k}}^{D}$ in the vertex (3.16) is related to the $\mathbf{k}$-dependence of the DDW gap in the GF (3.14). Using the definition (3.15) of the correlation function and adding an isotropic impurity scattering we evaluated in Ref. [12] the optical conductivity in the DDW state, see the inset of Fig. 4 . Here we report the $T=0$ value of the optical conductivity in a system without DDW and with DDW for $\delta=0.13$, using the same parameter values as in Fig. 3. As usual, when DDW order forms the optical conductivity presents both a Drude peak and an absorption at finite frequency $\sim 2 \mu$ due to the optical processes between the two quasiparticle branches $\xi_{ \pm}$(see also Ref. [31] for the case with $t^{\prime} \neq 0$ ). Although the Drude peak is reduced by the DDW formation, these interband finite-frequency processes lead to an overall increase of the spectral weight with respect to the case without DDW, as one can see in the main panel of Fig. 4 where we report $N(\omega)=2 \int_{0}^{\omega} \sigma\left(\omega^{\prime}\right) d \omega^{\prime}$. The crossing of $N^{D D W}$ with respect to $N$ at $\omega \sim 2 t$ is again an effect of the additional term proportional to $v_{D}$ in the current (3.7) and in the vertex (3.16), which extend above the Drude peak the effect of the DDW formation. Indeed, as discussed in detail in Ref. [12], and consistent with the results of Valenzuela et al. in Ref. [31], when the contribution proportional to $v_{D}$ is omitted one finds instead in the DDW state a spectral weight lower than in the normal state (in analogy with the behavior across the SC transition described by ordinary BCS theory).

\section{OPTICAL CONDUCTIVITY AND SUM RULE WITHIN A FULL MODEL WITH DDW INSTABILITY}

\section{A. Limitations of the reduced model and need of a more microscopic approach}

There is no doubt that the results presented above for the electric current operator (3.7), the optical conductivity shown in Fig. 4 and the sum rule (3.11) (and its generalization (3.13) valid in the superconducting state that forms on top of the DDW state) are consistent with each other, as far as they are considered within the framework of the reduced model (3.4). 


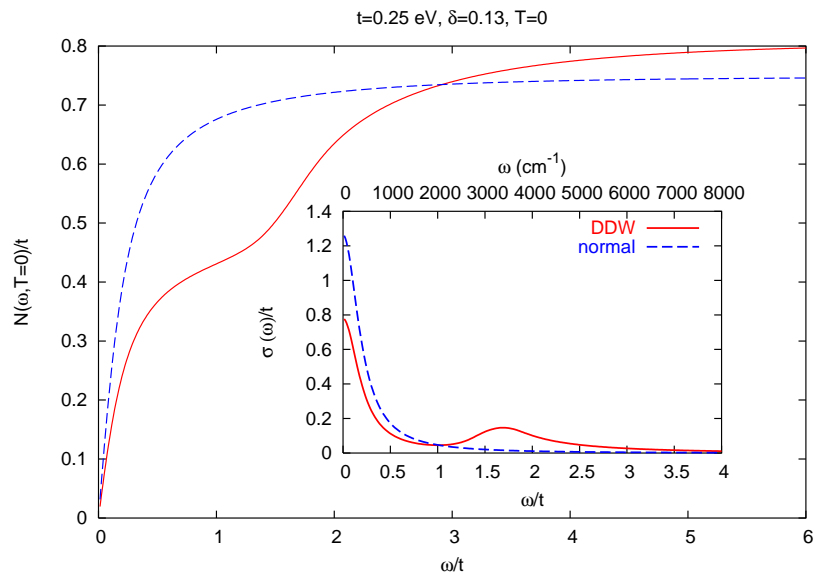

FIG. 4: Integrated spectral weight in units of $e^{2} \pi a^{2} / V$ for the optical conductivity at $T=0$ of a system with and without DDW. The values of parameters are the same as in Fig. 3. At low cut-off energy the spectral weight in the DDW state is lower than in the normal state, but when intraband excitations are taken into account the spectral weight of the DDW state exceeds the value of the normal state. Inset: optical conductivity in the two cases.

One could, however, still question these results from the more general point of view of the validity of the Hamiltonian (3.4), viz. one may question whether the low-energy Hamiltonian (3.4) is suitable for the description of the intraband excitations that cause the increase of $W^{D D W}(D, T)$ as $T$ decreases. The justification of the Hamiltonian (3.4) can only be made using a more microscopical Hamiltonian (2.1). In many ways this resembles the history of the reduced BCS Hamiltonian, since the theory of superconductivity was completed only when a more generic Hamiltonian was studied (see e.g. Refs. [17, 48]). These analogies between DDW and BCS models were exploited in Ref. [12]. One of the main conclusions of Ref. [12] is that the relation between the optical conductivity $\sigma(\omega)$ and the sum rule for the full model (2.1) can be established by looking at the problem of the vertex corrections in the current-current correlation functions. This vertex is determined by solving an integral equation, whose solution is a formidable problem. Its zero-frequency solution provided by the Ward identity was used in Ref. [12]. More recently Aristov and Zeyher [13] extended this analysis to finite frequency and investigated the behavior of sum rule in the full DDW model.

\section{B. Electrical conductivity and equation for vertex}

The current-current correlation function defined by Eq. (2.5) is expressed in terms of the DDW Green's function (3.14), the bare vertex $\gamma_{i}^{0}$ and the full vertex function $\Gamma_{j}$ as follows [17]:

$$
\Pi_{i j}\left(\mathbf{q}=\mathbf{0}, i \Omega_{m}\right)=-2 \frac{T}{N} \sum_{\mathbf{k}, i \omega_{n}}^{R B Z} \operatorname{Tr}\left[G\left(\mathbf{k}, i \omega_{n}\right) \gamma_{i}^{0}(\mathbf{k}) G\left(\mathbf{k}, i \omega_{n}+i \Omega_{m}\right) \Gamma_{j}\left(\mathbf{k}, i \Omega_{m}\right)\right] .
$$

where $\gamma^{0}$ and $\Gamma$ represent the bare and full vertex function, respectively. A careful reader may notice that the vertex $\gamma_{i}^{0}$ for a model based on the full Hamiltonian (2.1) is nothing else then Eq. (2.12). Using the matrix notation introduced in Sec. III it reads

$$
\gamma_{\mu}^{0}(\mathbf{k})=\left(v_{\mathbf{k}}^{F} \sigma_{3}, \sigma_{0}\right)
$$

Comparing the bare vertex $\gamma_{i}^{0}$ with the vertex $\gamma_{i}$ [see Eqs. (3.16) and (3.9)] of the reduced model, it becomes clear that the bare bubble approximation when the full vertex $\Gamma_{j}(\mathbf{k}, \omega)$ is replaced at will by the bare vertex $\gamma_{i}^{0}(\mathbf{k})$ would miss completely the physics of the problem. Thus one has to find the full vertex by solving the integral equation for it $[12,13]$

$$
\Gamma_{i}\left(\mathbf{k}, i \Omega_{m}\right)=\gamma_{i}^{0}(\mathbf{k})+T \sum_{x_{l}} \int d \mathbf{p} V(\mathbf{p}-\mathbf{k}) G\left(\mathbf{p}, i x_{l}\right) \Gamma_{i}\left(\mathbf{p}, i \Omega_{m}\right) G\left(\mathbf{p}, i x_{l}+i \Omega_{m}\right)
$$

where the current vertex $\Gamma_{i}$ is a $2 \times 2$ matrix and the integration over $\mathbf{p}$ with the measure $d \mathbf{p}=d p_{x} d p_{y} /(2 \pi)^{2}$ runs over the full (chemical) Brillouin zone, while $\mathbf{q}$ lies within the reduced zone. Here $V(\mathbf{p}-\mathbf{q})$ represents the Fourier 
transform of the potential $V\left(\mathbf{r}_{i}-\mathbf{r}_{j}\right)$ of Eq. (3.2). In Ref. [13] the interaction term of the Hamiltonian $H_{\text {int }}$ is chosen to be the Heisenberg interaction (3.1) between nearest neighbors, so that in the momentum representation $V(\mathbf{k})=J(\mathbf{k})=2 J\left(\cos k_{x} a+\cos k_{y} a\right)$. After analytical continuation $i \Omega_{m} \rightarrow \omega+i 0$ is made, one can consider the static limit $\omega=0$ of Eq. (4.3) to illustrate that if a vertex function $\Gamma_{i}$ satisfies the Ward identity

$$
\Gamma_{i}(\mathbf{k}, 0)=-\frac{\partial G^{-1}(\mathbf{k}, 0)}{\partial \mathbf{k}_{i}}
$$

it is also a solution of the equation (4.3) for vertex. The crucial assumption in this proof (see Refs. [12, 17]) is that the potential $V$ is nonseparable, viz. it depends on the difference $\mathbf{p}-\mathbf{k}$. The restrictions on the form of the potential $V(\mathbf{p}, \mathbf{k})$ necessary to satisfy the Ward identity (4.4) and, respectively, to obey the charge conservation law deserve special study (see e.g. Refs. [53, 54]). It was observed in Ref. [13] that an interesting feature of Eq. (4.3) is that, for the specific potential $J(\mathbf{p}-\mathbf{k})$ considered there, only the odd kernels (like $\sin p_{x} a \sin k_{x} a$ ) of its decomposition into the sum of separable kernels can contribute in the integral over $\mathbf{p}$, while these terms were absent in the reduced model (3.3). Finally, we note that the relation (4.4) was used above in Eq. (3.17), since for the reduced non-interacting model (3.4) it allows to obtain the exact vertex valid for all frequencies [12].

The equation for the vertex (4.3) with the potential $J(\mathbf{k}-\mathbf{p})$ written above is solved at finite frequency in Ref. [13]. The corresponding optical conductivity (2.8) calculated on the base of the full bubble (4.1) reads

$$
\sigma(\omega)=\frac{e^{2}}{\omega} \operatorname{Im} \int d \mathbf{k} \int d x_{1} d x_{2} \frac{n_{F}\left(x_{1}\right)-n_{F}\left(x_{2}\right)}{\omega-x_{1}+x_{2}+i 0} \operatorname{Tr}\left[\gamma_{i}(\mathbf{k}) A\left(\mathbf{k}, x_{1}\right) \Gamma_{i}(\mathbf{k}, \omega) A\left(\mathbf{k}, x_{2}\right)\right],
$$

where the integration is over RBZ and $2 \pi i A(\omega, \mathbf{k})=G_{A}(\omega, \mathbf{k})-G_{R}(\omega, \mathbf{k})$ is the spectral function expressed via the difference of advanced and retarded GF. Note that to regularize the electrical conductivity in the limit $\omega \rightarrow 0$ one has to consider the scattering by impurities which is included in the simplest form by introducing a finite quasiparticle lifetime, $\tau_{q p}$. The expression for $\sigma(\omega)$ becomes rather complicated when the solution of (4.3) is substituted there, but in the limits $\omega \rightarrow 0$ and $\omega \tau_{q p} \gg 1$ it can be significantly simplified. These two limits are considered below.

\section{Conductivity for $\omega \rightarrow 0$ and $\omega \tau_{q p} \gg 1$}

According to the results of Refs. [12,13], in the limit $\omega \rightarrow 0$ the conductivity $\sigma(\omega)$ reduces to the "naive" expression

$$
\sigma(\omega)_{\text {naive }}=\frac{e^{2}}{\omega} \operatorname{Im} \int d \mathbf{k} \int d x_{1} d x_{2} \frac{n_{F}\left(x_{1}\right)-n_{F}\left(x_{2}\right)}{\omega-x_{1}+x_{2}+i 0} \operatorname{Tr}\left[\Gamma_{i}(\mathbf{k}, 0) A\left(\mathbf{k}, x_{1}\right) \Gamma_{i}(\mathbf{k}, 0) A\left(\mathbf{k}, x_{2}\right)\right],
$$

with the static vertex $\Gamma_{i}(\mathbf{k}, 0)$. The dc limit of $\sigma(\omega)_{\text {naive }}$ was obtained also in Ref. [51], where the link between the present problem and the approach developed by Langer [55] was pointed out.

Comparing the LHS of Eqs. (4.4) and (3.17) which enter, respectively, in the current-current correlation function for DDW model (3.15) and in Eq. (4.6), one can notice that $\sigma(\omega)_{\text {naive }}$ coincides with the exact $\sigma(\omega)$ derived from Eq. (3.15) for the reduced DDW model. This coincidence was the main motivation for the work of Ref. [12], where the conductivity $\sigma(\omega)_{\text {naive }}$, which is just an approximation for the full model (2.1) with $H_{\text {int }}$ given by Eqs. (3.1), was extrapolated to higher $\omega$ and studied in detail.

The analysis of Ref. [13] clarifies that Eq. (4.6) does not remain valid for higher $\omega$. Considering the general shape of the optical conductivity depicted in Fig. 4, one finds that: (i) as far as the Drude peak is concerned, for $\omega \tau_{q p} \gg 1$ the current vertex $\Gamma_{i}(\mathbf{k}, \omega)$ changes from its $\omega=0$ value Eq. (4.4) to its bare value Eq. (4.2). Accordingly, the value of $\sigma(\omega)$ already near the tail of the Drude peak may well be estimated using Eq. (4.5) with two bare vertices $\gamma_{i}^{0}(\mathbf{k})$; (ii) as far as intraband transitions are concerned, the vertex correction enhance $\sigma(\omega)$ only near the edge of the intraband optical absorption. Thus the expression (4.6) overestimates the value of $\sigma(\omega)$ both in the Drude part, for $\omega \tau_{q p} \gg 1$, and in the region of intraband transitions. A somewhat counterintuitive result of Ref. [13] is that the crossover from the full vertex $\Gamma_{i}(\mathbf{k}, \omega)$ to the bare vertex $\gamma_{i}^{0}(\mathbf{k})$ occurs at $\omega \sim \tau_{q p}^{-1}$ rather than at the energy scale $D_{0}$ related to the formation of the DDW order. It would be interesting to study how this result depends on the choice of the microscopic $H_{\text {int }}$. We note also the influence of the full vertex $\Gamma_{i}(\mathbf{k}, \omega)$ discussed here is obtained under the assumption that the impurity scattering rate $1 / 2 \tau_{q p}$ is isotropic. As shown recently in Ref. [32], these results may change drastically if $1 / 2 \tau_{q p}$ acquires a $\mathbf{k}$-dependence.

\section{Behavior of the spectral weight}

The temperature dependence of the spectral weight $W(T)$ obtained in Ref. [13] turns out to be opposite to the behavior of $W(D, T)$ considered in Sec. III, viz. in the DDW state it decreases as the temperature $T$ decreases and 
the DDW gap $D(T)$ opens. This decrease follows from the fact that for $D_{0}>\tau_{q p}^{-1}$ the vertex $\Gamma_{i}(\mathbf{k}, \omega) \sim \gamma_{i}^{0}(\mathbf{k})$, so that the weight of intraband excitations is smaller than in the reduced model, where it is crucial for producing an increase of $W^{D D W}(D, T)$ that the vertex $\gamma_{i}(\mathbf{k})[56]$ is effective for all frequencies $\omega$ (see Sec. III). Observe that this result should be expected according to the fact that the kinetic energy increases when the DDW state is formed. As was explained in detail in Ref. [12], when vertex corrections are properly included in the current-current correlation function the resulting sum rule is given by Eq. (2.13) with a modified occupation number due to the DDW transition, so that $W(T)$ decreases, in analogy to what $W(\Delta, T)$ given by Eq. (2.24) does in the superconducting state.

The results of Ref. [13] also show that in the presence of the full vertex the absolute value of the spectral weight significantly increases with respect to the estimate made with the bare vertex $\gamma_{i}^{0}(\mathbf{k})$. Moreover, this increase is seen already in the normal state above $T_{D D W}$, so that one can consider that even when $W(T)$ starts to decrease below $T_{D D W}$, the absolute value of $W(T)$ is still well above the value of the weight estimated with the bare vertex $\gamma_{i}^{0}(\mathbf{k})$. This result should be contrasted with the analysis of Ref. [22, 30], where instead it was shown that the interaction decreases the absolute value of the normal-state spectral weight with respect to the non-interacting case, mainly as a consequence of the quasiparticle renormalization which is instead absent Ref. [13]. Thus, further investigation is required to understand whether this discrepancy is related simply to the choice of different microscopic models or it is caused by the approximations used to evaluate the spectral-weight behavior.

\section{OPEN QUESTIONS AND RELATIONSHIP TO OTHER SYSTEMS}

Our purpose here was not to convince our reader that the DDW state is the best candidate to explain the pseudogap behavior observed in HTSC. Neither the reduced nor the full model show full agreement with existing experimental optical-conductivity data, due in particular to the lack of a satisfactory description of the shape of the optical spectra observed experimentally. In particular, no clear signature of the interband processes characteristic of the DDW state has been observed in cuprates [20], even though some signatures of finite-frequency peaks have been reported recently [57], which can still be attributed to some kind of charge ordering [58]. Moreover, while the reduced DDW model shows at least an interesting feature that agrees with the experiments, i.e. an increasing spectral weight in the non $\mathrm{SC}$ state, an attempt to generalize it and consider a more microscopic model destroys this behavior. Certainly more work has to be done to understand the link between reduced and full models. Nevertheless we hope that the results discussed here for the restricted optical-conductivity sum rule in the DDW state may be useful for the investigation of this sum rule in more sophisticated models leading to unconventional charge-density wave ordering or circulating currents (see e.g. [39]). Since the self-energy in these models is also k-dependent, to study the transport properties consistently one should definitely include vertex corrections. Finally we mention that the results presented here may be useful not only to cuprates, where they can be only partly applied, but also to other materials displaying a true $k$-space modulated CDW, as for example organic conductors [43] and dichalcogenide materials [45, 46].

\section{ACKNOWLEDGMENTS}

We express our deep gratitude to N. Andrenacci, H. Beck, S. Caprara, C. Di Castro and V.P. Gusynin for fruitful collaboration on the topics discussed in this review. We also thank D. Aristov, J.P. Carbotte, J. Hwang, V.M. Loktev, M. Ortolani, A. Toschi, D. Van der Marel for helpful discussions and careful reading of the manuscript. This work was supported by the Natural Science and Engineering Council of Canada (NSERC) and by the Canadian Institute for Advanced Research (CIAR).

[1] H.J.A. Molegraaf, C. Presura, D. van der Marel, P.H. Kes, M. Li, Science 295, 2239 (2002).

[2] A.F. Santander-Syro, R.P.S.M. Lobo, N. Bontemps, Z. Konstantinovic, Z.Z. Li, H. Raffy, Phys. Rev. Lett. 88, 097005 (2002); Europhys. Lett. 62, 568 (2003).

[3] A.F. Santander-Syro, R.P.S.M. Lobo, N. Bontemps, W. Lopera, D. Girata, Z. Konstantinovic, Z.Z. Li, H. Raffy, Phys. Rev. B 70, 134504 (2004).

[4] C.C. Homes, S.V. Dordevic, D.A. Bonn, R. Liang, W.N. Hardy, Phys. Rev. B 69, 024514 (2004).

[5] A.V. Boris, N.N. Kovaleva, O.V. Dolgov, T. Holden, C.T. Lin, B. Keimer, C. Bernhard, Science 304, 708 (2004).

[6] M. Ortolani, P. Calvani, S. Lupi, Phys. Rev. Lett. 94, 067002 (2005).

[7] G. Deutscher, A.F. Santander-Syro, N. Bontemps, cond-mat/0503073.

[8] F. Carbone, A.B. Kuzmenko, H.J.A. Molegraaf, E. van Heumen, E. Giannini, D. van der Marel, preprint 2005.

[9] A.B. Kuzmenko, H.J.A. Molegraaf, F. Carbone, D. van der Marel, cond-mat/0503768. 
[10] A. F. Santander-Syro, N. Bontemps, cond-mat/0503767.

[11] L. Benfatto, S. Sharapov, and H. Beck, Eur. Phys. J. B 39, 469 (2004).

[12] L. Benfatto, S. Sharapov, N. Andrenacci and H. Beck, Phys. Rev. B 71104511 (2005).

[13] D.N. Aristov and R. Zeyher, cond-mat/0504633.

[14] A.J. Millis, Strong Interactions in Low Dimensions, ed. by D. Baeriswyl and L. De Giorgi, Kluver Accad. Pub, 2003.

[15] G. D. Mahan, Many-Particle Physics, Kluwer Acad. Pub., New York, 2000.

[16] D.J. Scalapino, S.R. White, and S. Zhang, Phys. Rev. B 47, 7995 (1993).

[17] J.R. Schrieffer, Theory of superconductivity, Addison Wesley (1988).

[18] J. Jaklič and P. Prelovšek, Adv. Phys. 49, 1 (2000).

[19] D. N. Basov and T. Timusk, Rev. Mod. Phys. 77, 721, (2005).

[20] T. Timusk, Sol. St. Comm. 127, 337 (2003).

[21] T. Timusk and B. Statt, Rep. Progr. Phys. 62, 61 (1999).

[22] A. Toschi, M. Capone, M. Ortolani, P. Calvani, S. Lupi, C. Castellani, Phys. Rev. Lett. 95, 097002 (2005).

[23] See for example J. L. Tallon, Phys. Rev. B 68, 180501 (2003) and references therein.

[24] J.E. Hirsch, and F. Marsiglio, Phys. Rev. B 62, 15131 (2000).

[25] M.R. Norman, and C. Pépin, Phys. Rev. B 66, 100506 (2002).

[26] T.K. Kopeć, Phys. Rev. B 67, 014520 (2003).

[27] T. Eckl, W. Hanke, and E. Arrigoni, Phys. Rev. B 68, 014505 (2003).

[28] V.M. Loktev, R.M. Quick, S.G. Sharapov, Phys. Rep. 349, 1 (2001).

[29] A. Knigavko, J.P. Carbotte, and F. Marsiglio, Phys. Rev. B 70, 224501 (2004).

[30] E. Schachinger and J.P. Carbotte, Phys. Rev. B 72, 014535 (2005).

[31] B. Valenzuela, E.J. Nicol, and J.P. Carbotte, Phys. Rev. B 71, 1334503 (2005).

[32] R. Gerami and C. Nayak, cond-mat/0506237.

[33] S. Chakravarty, R.B. Laughlin,D.K. Morr, and C. Nayak, Phys. Rev. B 63, 094503 (2001).

[34] B.I. Halperin, and T.M. Rice, Sol. State Phys. 21, 115 (1968).

[35] I. Affleck, J.B. Marston, Phys. Rev. B 37, 3774 (1988).

[36] J.B. Marston, I. Affleck, Phys. Rev. B 39, 11538 (1989).

[37] A.A. Nersesyan and G.E. Vachanadze, J. Low Temp. Phys. 77, 293 (1989).

[38] H.J. Schulz, Phys. Rev. B 39, 2940 (1989).

[39] C.M. Varma, Phys. Rev. B 55, 14554 (1997); preprint cond-mat/0507214.

[40] M.V. Eremin, I.A. Larionov, Pis'ma Zh. Eksp. Teor. Fiz. 68, 583 (1998) [JETP Lett. 68, 611 (1998)].

[41] E. Cappelluti and R. Zehyer, Phys. Rev. B 59, 6475 (1999).

[42] L. Benfatto, S. Caprara, and C. Di Castro, Eur. Phys. J. B 17, 95 (2000).

[43] B. Dóra, K. Maki, and A. Virosztek, Mod. Phys. Lett. B 18, 327 (2004); H. Won, S. Haas, K. Maki, D. Parker, B. Dora, A. Virosztek, cond-mat/0508234.

[44] C. Castellani, C. Di Castro, and M. Grilli, Phys. Rev. Lett. 75, 4650 (1995); C. Di Castro, L. Benfatto, S. Caprara, C. Castellani, M. Grilli, Physica C 341-348, 1715 (2000).

[45] V. Vescoli, L. Degiorgi, H. Berger and L. Forró, Phys. Rev. Lett. 81, 453 (1998).

[46] A. H. Castro Neto, Phys. Rev. Lett. 86, 4382 (2001).

[47] D.N. Aristov and R. Zeyher, cond-mat/0405194, SCES04 proceedings; Phys. Rev. B 70, 212511 (2004).

[48] Y. Nambu, Phys. Rev. 117, 648 (1960).

[49] H.-Y. Kee and Y.B. Kim, Phys. Rev. B 66, 052504 (2002).

[50] X. Yang and C. Nayak, Phys. Rev. B 65, 064523 (2002).

[51] S.G. Sharapov, V.P. Gusynin, and H. Beck, Phys. Rev. B 67, 144509 (2003).

[52] Note that the arguments of the vertex functions here are written using the conventions of Ref. [13] which are different from Ref. [12].

[53] D. Vollhardt and P. Wölfle, Phys. Rev. B 22, 466 (1980).

[54] H.T. Nieh, P. Sheng, and X.-B. Wang, Phys. Lett. A 246, 522 (1998).

[55] J.S. Langer, Phys. Rev. 127, 5 (1962)

[56] Recall that for the reduced DDW Hamiltonian this vertex and the corresponding $\sigma(\omega)$ are exact.

[57] A. Lucarelli, S. Lupi, M. Ortolani, P. Calvani, P. Maselli, M. Capizzi, P. Giura, H. Eisaki, N. Kikugawa, T. Fujita, M. Fujita, and K. Yamada Phys. Rev. Lett. 90, 037002 (2003); C. Bernhard, T. Holden, A. V. Boris, N. N. Kovaleva, A. V. Pimenov, J. Humlicek, C. Ulrich, C. T. Lin, and J. L. Tallon, Phys. Rev. B 69, 052502 (2004).

[58] L. Benfatto and C. Morais Smith, Phys. Rev. B 68, 184513 (2003). 